\title{
Wi_Fi Positioning Utilizing Triangulation Strategies and AoA In Indoor Environments
}

\author{
Zahraa A. Hamza ${ }^{1}$, Mahmood F Mosleh ${ }^{1}$, Faeza A. Abed ${ }^{2}$ \\ \{ Zahraaabbasct9@gmail.com ${ }^{1}$,drmahmoodfarhan@gmail.com ${ }^{2}$, \\ dr.faeza.khafaji@gmail.com $\left.{ }^{3}\right\}$
}

\begin{abstract}
Middle Technical University, Electrical Engineering Technical College, Baghdad, Iraq ${ }^{\text {, }}$ Middle Technical University- Institute of Technology- Baghdad ${ }^{2}$
\end{abstract}

\begin{abstract}
Implementing a limiting framework for an indoor climate has been complicated by multipath and blockage difficulties. The Angle of Arrival (AoA) is the most commonly used method for such situations in the area, and it should be strengthened, especially for additional obstructions due to massive multipath issues. A contextual investigation of a chosen fabricating is chosen in this paper to use the offered approach of this analysis. This concept is based on the triangulation approach. The Wireless Insite Software (WIS) was used to reconstruct the contextual analysis territory, with 30 Receivers (RXs) and 2 Transmitters (TXs) placed in appropriate locations. Following the estimation of AoA and saving them into the database, the coordinates of RXs are determined using MATLAB programming. The findings show that the estimated locations are reasonably near to the genuine locations, with an average error of $(0.24)$ metre for the $\mathrm{X}$-coordinate and (0.205857) metre for the Y-coordinate. As a result, we find that under the Triangulation method, the AoA approach is more effective in evaluating indoor localisation.
\end{abstract}

Keywords: WIS, AoA, Triangulation.

\section{Introduction}

As the popularity of mobile devices such as smartphones, smart watches, laptops, and some connected devices has grown, so has the number of Location-Based Services (LBS) available to assist us in areas such as emergency services, navigation, childcare, geophone, social networking, entertainment/gaming, asset monitoring, and so on [1]. Clients with smartphones, for example, can easily plan their trips using Google Maps and hail a nearby cab. The Global Positioning System (GPS) essentially determines the exact area (GPS). However, the GPS signal is too severely degraded to be obtained in inside situations. To meet the growing need for indoor LBS applications, a variety of solutions have been developed [2]. In contrast to Line-of-Sight (LoS) limitation in outdoor environments, where worldwide route satellite frameworks are for all intents and purposes, the default arrangement presents advances for indoor environments in Non-Line-of-Sight (NLoS) conditions have not united to a universally perceived arrangement [3]. Consequently, this subject draws in broad exploration interest [4]. The primary objective in NLoS conditions is to utilize the all-around sent earthbound innovations and concentrate data from the got radio signs, like Received Signal Strength (RSS) [5], Time of Arrival (ToA) [6], AoA [7], Time Difference of Arrival (TDoA) [8], Phase Difference of Arrival (PDoA) [9] or a hybrid of them [10]. As usual, indoor conditions are convoluted, so some difficulties and impediments impact the exactness of gadgets. In the instance of NLoS, this impact happens when articles recognize senders and collectors [11]. The example of transmission assumes a 
huge part in the estimate. The got signal at that point enters the beneficiary from dividers or different articles, hence the regard of the ToA from the association with another. The other consequence weakens as recipients respond to a weak signal, making it difficult to determine the proper area based on this signal [12]. To overcome these challenges, the analysts suggested a variety of solutions for increasing gadget productivity by limiting the effects of NLoS and other constraints. NLoS identification [13], or the unique mark technique advocated in a few studies, such as [14], to the estimated specific RSS esteem, is one of these options. The fact that triangulation is proposed for assessing localizability in this study. Following that, by iteratively applying Triangulation to a network, it is possible to find localizable hubs. Triangulation is widely used in practise [15] since it is adaptable, simple to execute, and effective in terms of correspondence and computation [16]. The following is a breakdown of the content of this document. The indoor localization theory is presented in the second section. The details of the case study building are detailed in section three. The proposed approach's results and analysis are reported in section four. Finally, in part five, the conclusion is presented.

\section{Indoor localization theory}

There are various strategies for tending to data with respect to the space of the position. A rudimentary sort of spatial portrayal is a mathematical depiction. A graphical diagram depicts the space with its mathematical structure and a collection of coordination focuses under a Cartesian arrange reference plot. The space model has a strong representation that is commonly used with directions to communicate a theoretical space, such as (X, Y, Z). Truly, a room describes by various mathematical structures. A mathematical structure surely is a crude structure 2D or 3D. Specifically, executions, the type of development can be applied to more perplexing structures. Directions are picked and requested for each space in an unmistakable construction regarding the mathematical type of room. The meaning of directions is carefully associated with the mathematical type of room. Utilizing the crucial ideas of the convergence of different lines and the privileged mathematical detailing to rough the heading of a hub, the specific point in the situating area strategies can be resolved. The position directions of the hubs are determined from numerous points of view, like AoA, ToA, TDoA, and RSS. To gauge the course places of a hub utilizing lines and points, these methods depended on mathematical standards. Instances of these ideas are Triangulation, trilateration, and exaggerated [17].

\subsection{Triangulation technique}

The Triangulation situating the area method utilizes the mathematical properties of triangles to assess a hub situating. This standard comprises three focuses, two reference focuses $\left(\mathrm{Z}_{1}\right.$ and $\mathrm{Z}_{2}$ ), and an objective point $(\mathrm{T})$. At the crossing point of the line from $\mathrm{Z}_{1}$ to $\mathrm{T}$ and from $Z_{2}$ to $T$, we get the two points $\Theta_{1}$ and $\Theta_{2}$ as demonstrated in Figure 1, and geometrical capacities are utilized to compute the objective position [18]. 


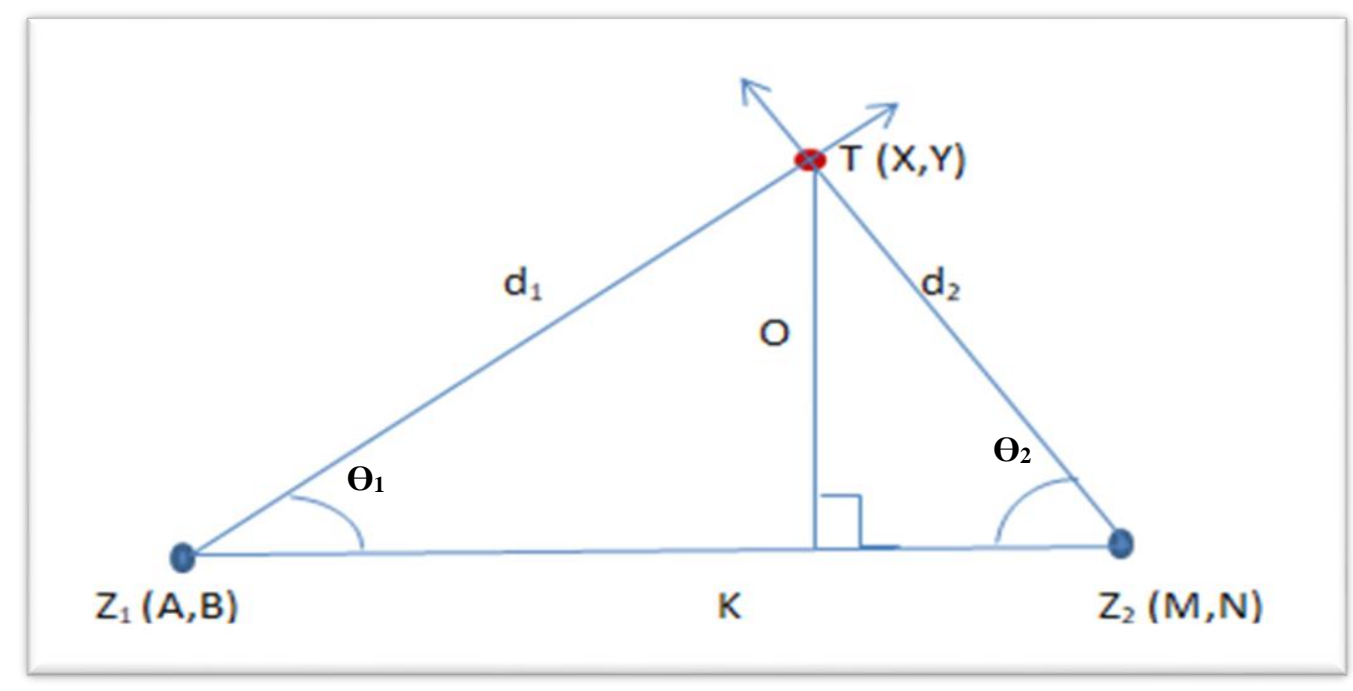

Fig. 1. Triangulation guideline.

$$
\begin{gathered}
K=\frac{o}{\tan \theta_{1}}+\frac{o}{\tan \theta_{2}} \\
O=\frac{K \cdot \sin \theta_{1} \cdot \sin \theta_{2}}{\sin \left(\theta_{1}+\theta_{2}\right)} \\
\theta_{1}=\tan ^{-1}\left(\frac{B-Y}{A-X}\right) \\
\theta_{2}=\tan ^{-1}\left(\frac{N-Y}{M-X}\right)
\end{gathered}
$$

Where $\mathrm{K}$ is the line that connects the reference focuses $\mathrm{Z} 1$ and $\mathrm{Z} 2$, and $\mathrm{O}$ is the line that connects target $\mathrm{T}$ and line $\mathrm{K}$. $\mathrm{d} 1$ is the line that connects a target point $\mathrm{T}$ to a reference point $\mathrm{Z} 1$, and $\mathrm{d} 2$ is the line that connects a target point $\mathrm{T}$ to a reference point $\mathrm{Z} 2 . . \mathrm{Z}_{2}, \Theta_{1}$ is the point between line $\mathrm{d} 1$ and line $\mathrm{K}, \mathrm{O}_{2}$ is the point between line $\mathrm{d}_{2}$ and line $K,(A, B)$ are the directions of the reference point $Z_{1}$ and $(M, N)$ are the directions of the reference point $Z_{2},(X, Y)$ are the directions of the target $T$.

The directions of the objective $(X, Y)$ can be determined as referenced beneath:

$$
\begin{aligned}
& X=\frac{N-B-M \tan \theta_{2}+A \tan \theta_{1}}{\tan \theta_{1}-\tan \theta_{2}} \\
& Y=N-M \tan \theta_{2}+X \tan \theta_{2}
\end{aligned}
$$

To discover the distances between focuses $Z_{1}, Z_{2}$, and the target point $T$, we utilize the following conditions [19].

$$
d_{-} 1=\sqrt{ }\left((A-X)^{\wedge} 2+(B-Y)^{\wedge} 2\right)
$$




$$
d_{-} 2=\sqrt{ }\left((M-X)^{\wedge} 2+(N-Y)^{\wedge} 2\right)
$$

\subsection{Triangulation technique based on AoA method}

AoA is an organization-based situating area strategy that actions the time take by radio signs to show up at a few focuses. AoA technique utilizes a receiving wire containing a different component cluster in which the specific area of each AoA component is known definitely. Every component is little and fit for getting a different sign. By estimating signal strength, the season of appearance, and the stage at every cluster component, it is feasible to figure the view way from the transmitter to the recipient. Putting another collector with a similar radio wire design in an alternate area permits rehashing the cycle. The convergence point of the two view ways addresses the area of the sending hub. The AoA technique requires at least two reference stations with directional radio wires with thin bar widths. This strategy is not difficult to carry out and is utilized in specific applications like reviewing, finding the wellspring of an unlawful transmitter, and following objective hubs. The AoA strategy utilizes the basic triangulation rule to figure the area of an objective hub and depends on vector running. The technique needs, at any rate, two reference stations to quantify the course of the got radio signs. The objective hub addresses the place of the crossing point of lines attracted from reference stations to the objective hub. A couple of vectors structure a solitary point in a moment that they cross with one another. The point of appearance figuring and other important data can be utilized to ascertain the situation of an objective hub. Handset stations with reference to arranging should be utilized as a reference to get exact figurings of the necessary data in this strategy. The data about AoA can be estimated in three different ways. The main sort dependent on estimating the got signal stage contrasts between the reception apparatus exhibit components. The second is to quantify a pillar shaping or ghostly thickness across the radio wire cluster of the recipient. The third method of assessing the situation of an objective gadget; to discover AoA is by utilizing sectored multipillar reception apparatuses. This technique utilized in cell networks that were at that point received cell-sectoring in the organization to accomplish more solid interchanges [20].

Allow us to assume we have a communicated signal from the target hub, organized (X, Y) to $i^{\text {th }}$ handset station, which coordinates $\left(\mathrm{X}_{\mathrm{i}}, \mathrm{Y}_{\mathrm{i}}\right)$. The line among the transmitter and the reference point converge with X-pivot makes a point $\Theta_{i}$ as demonstrated in Figure 2, this point will be the point of appearance which is characterized as:

$$
\tan \Theta_{i}=\frac{Y-Y_{i}}{X-X_{i}}
$$




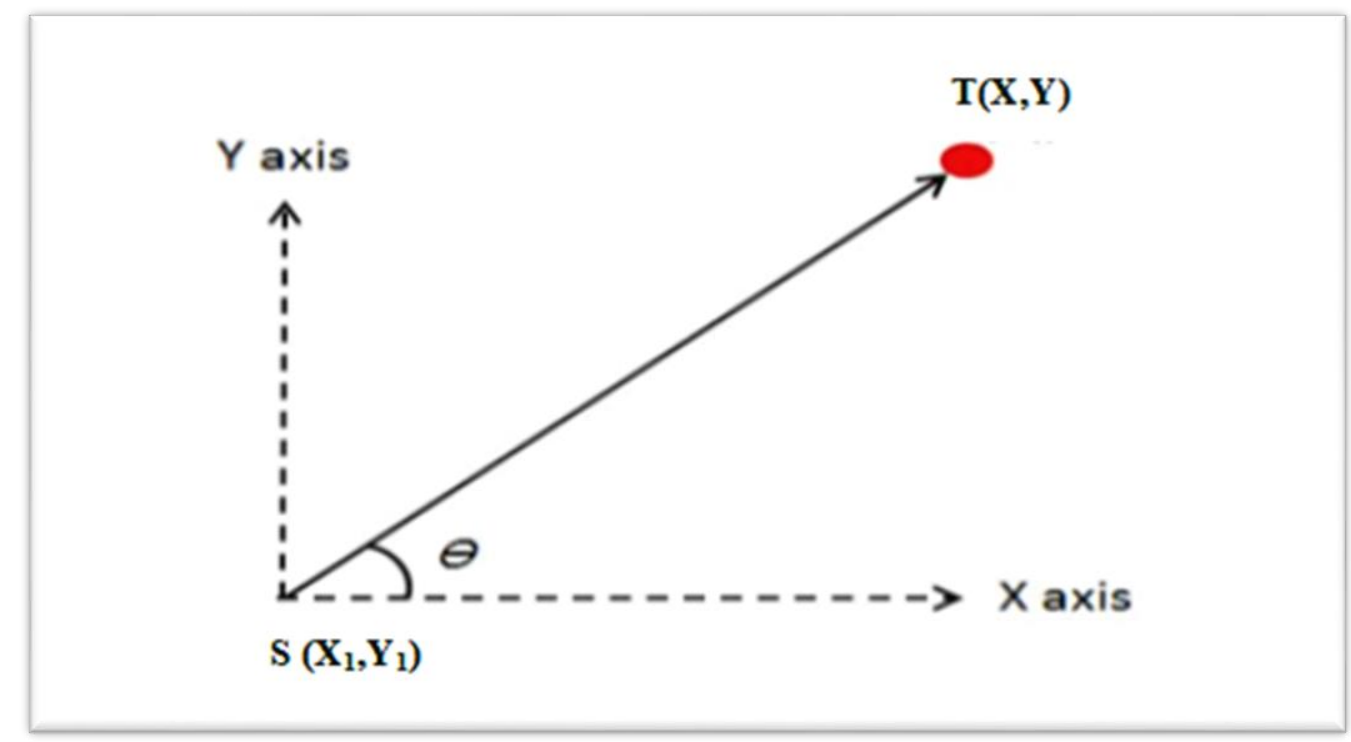

Fig. 2. The AoA theory.

To decide the directions of the target hub $\mathrm{T}$, we can utilize the accompanying conditions [21]:

$$
\begin{gathered}
X=\frac{K \tan \left(\theta_{2}\right)}{\tan \left(\theta_{2}\right)-\tan \left(\theta_{1}\right)} \\
Y=\frac{K \tan \left(\theta_{2}\right) \tan \left(\theta_{1}\right)}{\tan \left(\theta_{2}\right)-\tan \left(\theta_{1}\right)}
\end{gathered}
$$

Where $K$ is the distance between the reference stations $\mathrm{Z}_{1}$ and $\mathrm{Z}_{2}, \theta_{1}$ is the point of landing in the reference hub $\mathrm{Z}_{1}, \theta_{2}$ is the point of landing in the reference hub $\mathrm{Z}_{2},(X, Y)$ are the directions of the target hub T. Figure 3 shows the AoA technique. 


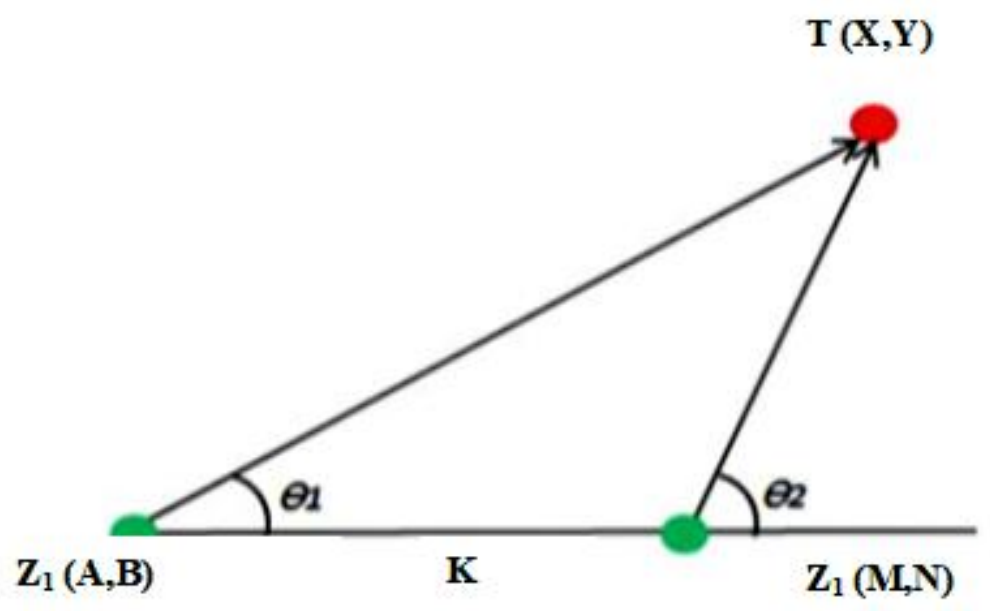

Fig. 3. AoA technique.

\section{Design case study building}

The EE Technical Building Campus decided to use the indoor localization metyhoology. A framework like this was created in simulation and experimental settings; the model was based on WIS. In the side half of the simulation, such a programme can calculate relevant parameters. The experimental data gathered using the WIS programme will represent the characteristics of AoA measurement and utilising the MATLAB programme to determine the coordinates of RXs in the experimental side section.

\subsection{Simulation Process}

WIS-based architecture in three dimensions (3D), conisdfering true model. In addition to the two places for TXs, thirty positions were chosen for RX deployment to measure AoA, which were separated into 10 groups as shown in Figure 4. 


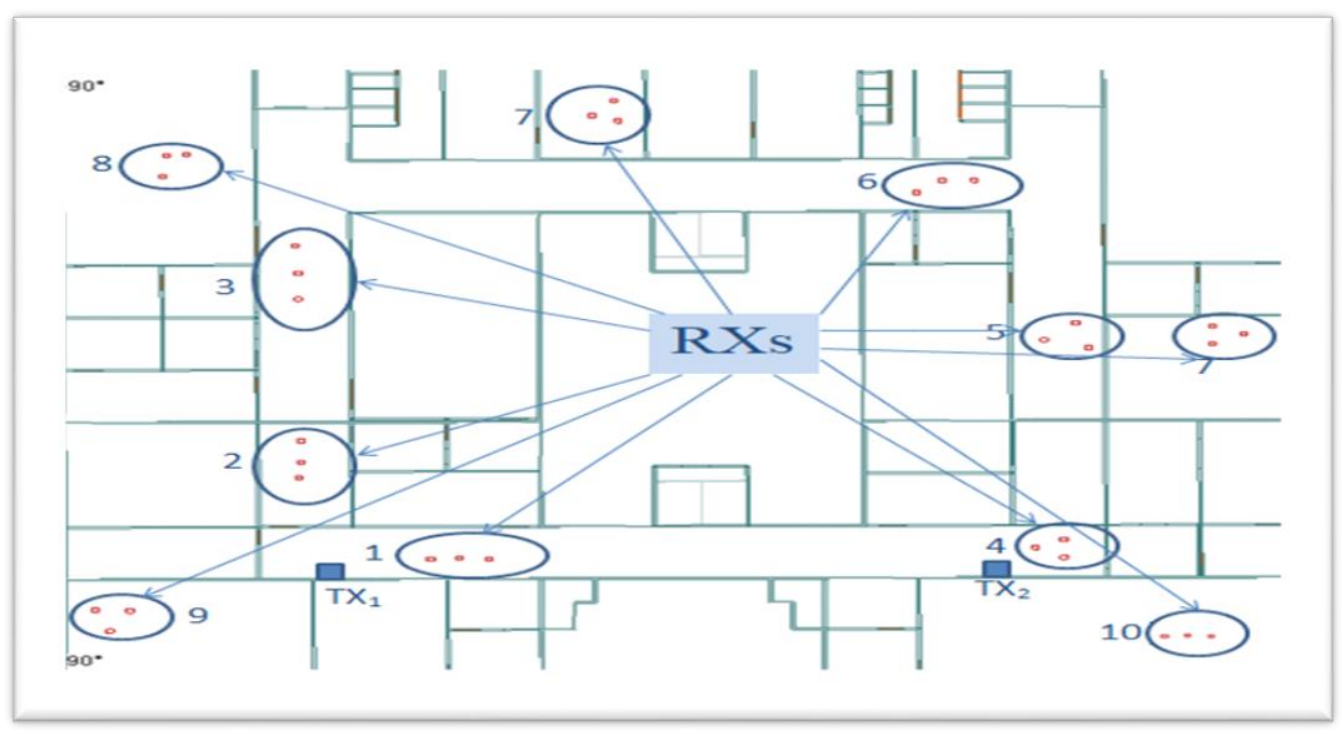

Fig. 4. Model of case study.

The transmitter and receiver parameters in WIS can be changed to fit the experimental devices, allowing the TXs and RXs mentioned in Table 1 to be assigned. All TXs and RXs are Omni-Directional to one experimental antenna, and the operational frequency is $2.4 \mathrm{GHz}$.

Table 1. The antennas properties

\begin{tabular}{lcc}
\hline Properties of antennas & TX & RX \\
\hline Antennas type & Omni.D. & Omni.D. \\
Input Power $(\mathrm{dBm})$ & 12.5 & - \\
Gain $(\mathrm{dBi})$ & 7.5 & 2.2 \\
E-Plane HPBW & $13^{\circ}$ & $94^{\circ}$ \\
Waveform & Sinusoid & Sinusoid \\
Temp. (k) & 296 & 296 \\
Polarizations & $\mathrm{V}$ & $\mathrm{V}$ \\
Received Threshold $(\mathrm{dBm})$ & -150 & -150 \\
\hline
\end{tabular}

The configuration of all materials' Permittivity and Conductivity to deal with actual ones is one of the key features of WIS. There are constant parameters to apply, as seen in table 2 and table 3. Based on such factors, conductivity and permittivity are calculated for each material. According to (), the conductivity denoted by () can be calculated as follows [22]:

$$
\sigma=c \times f^{d}
$$

Table 2 illustrates the constants $\mathrm{c}$ and $\mathrm{d}$. The Permittivity () is calculated in the following way:

$$
\eta=a \times \mathrm{f}^{\mathrm{b}}
$$


The constant $a$ and $b$ are related to each material used to construct the chosen building, which is listed in Table 3.

Table 2. The constant ( $c$ and $d$ ) is used in (12).

\begin{tabular}{lll}
\hline Material type & \multicolumn{1}{c}{$c$} & $\boldsymbol{d}$ \\
\hline Concrete & 0.0326 & 0.8095 \\
Brick & 0.038 & 0 \\
Wood & 0.0047 & 1.0718 \\
Glass & 0.0043 & 1.1925 \\
& & \\
\hline
\end{tabular}

Table 3. The constant ( $a$ and $b$ ) is used in (13).

\begin{tabular}{lcl}
\hline Material type & $\boldsymbol{a}$ & $\boldsymbol{b}$ \\
\hline Concrete & 5.31 & 0 \\
Brick & 3.75 & 0 \\
Wood & 1.99 & 0 \\
Glass & 6.27 & 0 \\
& & \\
\hline
\end{tabular}

As previously indicated, the $2.4 \mathrm{GHz}$ frequency employed based on equns (12) and (13). (13). The Permittivity and Conductivity are shown in Table 4 for reference frequency. The values in Table 4 will be set out in the WIS for materials used in building construction.

Table 4. Materials at 2.4GHZ.

\begin{tabular}{lcc}
\hline Material type & $\boldsymbol{\sigma}$ & $\boldsymbol{\varepsilon}$ \\
\hline Concrete & 0.0926 & 5.31 \\
Brick & 0.1079 & 3.75 \\
Wood & 0.0134 & 1.99 \\
Glass & 0.0122 & 6.27 \\
& & \\
\hline
\end{tabular}




\subsection{Side of experiment}

On the side of experimentation, Figure 5 shows the coordinates of the RXs extracted using WIS data for AoA in the MATLAB algorithm.

The effectiveness of measures to deal with practical measurements in the complete building improves in diverse contexts by taking various places in the case study building. In the case study context, two points were employed to empirically measure AoA with 30 samples each. The measured data are included in the database alongside the other measurements from the other two sites.

Finally, using the triangulation approach, the MATLAB programme supplied to convert the best data was recorded in the database to distance lead to RX coordinates.

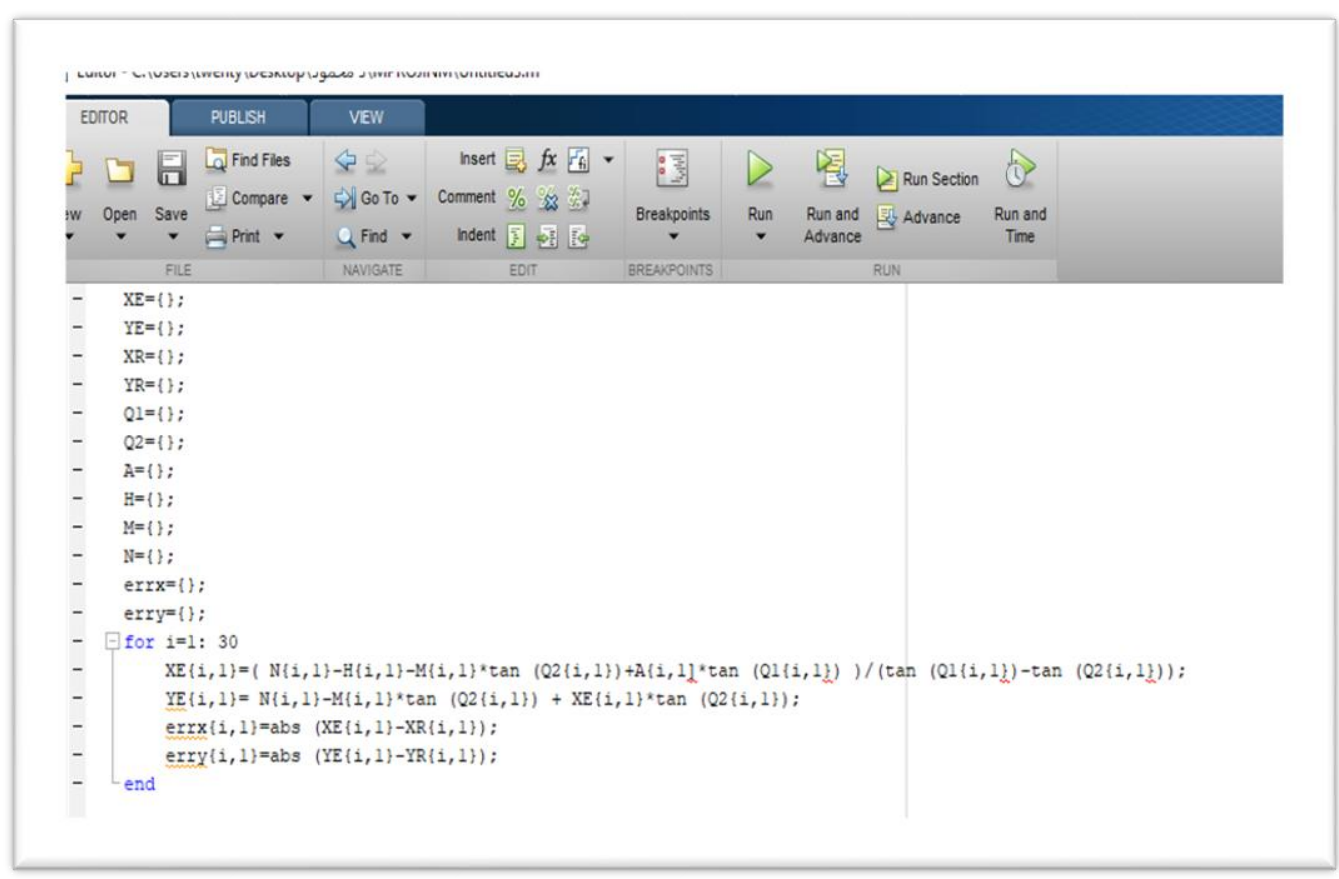

Fig. 5. Sample of MATLAB code.

\section{Results and analysis}

The approximate distance was calculated using AoA measurements; therefore, accurate AoA measurements would result in accurate distance calculation. The AoA method's error ratio between the real and estimated coordinates is shown in Figure 6, where the $\mathrm{X}$-maximum ratio error is 0.9 metre and the $\mathrm{Y}$-error ratio is 0.700262 metre. The error ratio for the $\mathrm{X}$-lowest coordinate is 0.02 metre, while the $\mathrm{Y}$-coordinates are 0.001643 metre. These findings are matched to those of [23]. The results obtained in such a reference said that ToA is superior to RSS and that inaccuracies (0.48-1.52) metres have been detected. Furthermore, the researcher [24] used $\mathrm{min} / \mathrm{max}$ positioning based on static ToA and RSS readings. When compared to our results, which show an average error of $(0.24)$ metre for X-coordinate and $(0.205857)$ metre for 
Y-coordinate based on AoA, the biconical antennas were installed with a frequency range of 3$11 \mathrm{GHz}$ in order to apply the trilateration technique, which gives better results than the $\mathrm{min} / \mathrm{max}$ method with a range of errors between (0.21-2.09) metre.

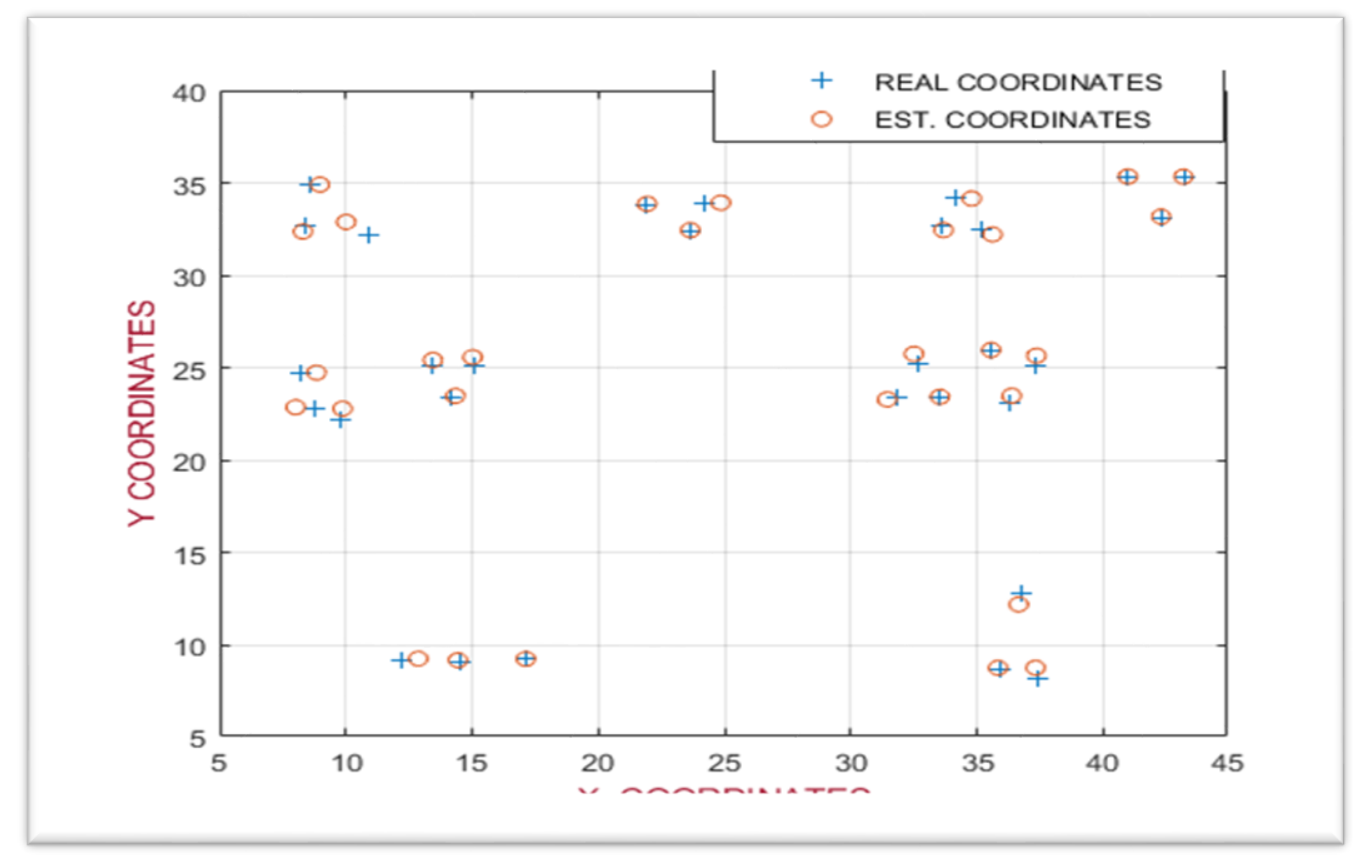

Fig. 6. AoA coordinates.

\section{Conclusions}

In this paper, with AoA methods, a proposed Triangulation strategy is completed to discover target areas in 10 positions conveyed in a choice building. These structures have been built to ascertain AoA dependent on WIS. In an appropriate area inside the contextual investigation building, two $2.4 \mathrm{GHz}$-based TXs were conveyed. On the trial line, WIS was used to measure AoA in two TXs circumstances, each with $30 \mathrm{RXs}$ cases. The outcomes reveal that a significant reduction in blunder (determined as the contrasts between the dramatic and recreated AoA). The average error for AoA $(0.24,0.205857)$ metre coordinates is shown as a result of our investigation. As a result of the the AoA strategy is effective than other techniques in computing indoor localisation.

\section{References}

[1] Roba Abbas, Katina Michael, MG Michael. The regulatory considerations and ethical dilemmas of location-based services (LBS): A literature review. Information Technology $\backslash \&$ People. 2014; 27 (1): 2-20.

[2] Gautam M. Borker, A. R. Mahajan. A review on propagation of secure data, prevention of attacks and routing in mobile ad-hoc networks (MANETs). International Journal of Data Science. 2018; 3(4): 353-389. 
[3] Robert F. Keefe, Ann M. Wempe, Ryer M. Becker, Eloise G. Zimbelman, Emily S. Nagler, Sophie L. Gilbert, Christopher C. Caudill. Positioning Methods and the Use of Location and Activity Data in Forests. Forests. 2019; 10(5): 458.

[4] M. M. Abdulwahid, O. A. Shareef Al-Ani, M. F. Mosleh, R. A. Abd-Alhmeed. A Comparison between Different C-band and mmWave band Frequencies for Indoor Communication. Journal of Communications. 2019; 41(10): 892-899.

[5] Yogita Chapre, Prasant Mohapatra, Sanjay Jha, Aruna Seneviratne. Received signal strength indicator and its analysis in a typical WLAN system. 38th Annual IEEE Conference on Local Computer Networks; 2013; Sydney, NSW, Australia. IEEE; 2013. P. 304-307.

[6] Ismail Guvenc, Chia-Chin Chong. A Survey on TOA Based Wireless Localization and NLOS Mitigation Techniques. IEEE Communications Surveys $\ \&$ Tutorials. 2009; 11(3): 107-124.

[7] Igor Dotlic, Andrew Connell, Hang Ma, Jeff Clancy, Michael McLaughlin. Angle of arrival estimation using decawave DW1000 integrated circuits. 2017 14th Workshop on Positioning, Navigation and Communications (WPNC); 2017; Bremen, Germany. IEEE; 2017. P. 1-6.

[8] Venkata B. M., R. K. Jeyachitra. Low Complexity Indoor Positioning System with TDOA Algorithm Using Hilbert Transform Method. Advances in Electrical and Computer Technologies; Singapore; Springer, 2020. p. 667-679.

[9] Yongtao Ma, Bobo Wang, Shuyang Pei, Yunlei Zhang, Shuai Zhang, Jiexiao Yu. An Indoor Localization Method Based on AOA and PDOA Using Virtual Stations in Multipath and NLOS Environments for Passive UHF RFID. IEEE Access. 2018; 6(1): 31772-31782.

[10] Sheng Zhang, Pengfei Du, Chen Chen, Wen-De Zhong, Arokiaswami Alphones. Robust 3D Indoor VLP System Based on ANN Using Hybrid RSS/PDOA. IEEE Access. 2019; 7(1): $47769-$ 47780.

[11] Feng Lyu, Nan Cheng, Hongzi Zhu, Haibo Zhou, Wenchao Xu, Minglu Li, Xuemin Sherman Shen. Intelligent context-aware communication paradigm design for IoVs based on data analytics. IEEE Network. 2018; 32(6): 74-82.

[12] Sun Ji-Ping, LI Chen-Xin. TOA underground coal mine target positioning method based on WiFi and timing error suppression. Journal of China Coal Society. 2014; 39(1): 192-197.

[13] Chen Huang, Andreas F. Molisch, Ruisi He, Rui Wang, Pan Tang, Bo Ai, Zhangdui Zhong. Machine learning-enabled LOS/NLOS identification for MIMO systems in dynamic environments. IEEE Transactions on Wireless Communications. 2020; 19(6): 3643-3657.

[14] Chavalit Koweerawong, Komwut Wipusitwarakun, Kamol Kaemarungsi. Indoor localization improvement via adaptive RSS fingerprinting database. The International Conference on Information Networking 2013 (ICOIN); 2013; Bangkok, Thailand. IEEE; 2013. P. 412-416.

[15] Wiegert, Roy F. Magnetic anomaly surveillance system using spherical trilateration. United States: Google Patents; 2013. 1-18.

[16] Khelifi, Fekher, et al. A survey of localization systems in the internet of things. Mobile Networks and Applications. 2019; 24(3): 761-785.

[17] Burrough, Peter A ,McDonnell, Rachael , McDonnell, Rachael A , Lloyd, Christopher D. Principles of geographical information systems. $3^{\text {rd }}$ Edition. United Kingdom. Oxford university press: 2015. 1-315.

[18] Luka Batistić, Mladen Tomic. Overview of indoor positioning system technologies. 2018 41st International Convention on Information and Communication Technology, Electronics and Microelectronics (MIPRO); 2018; Opatija, Croatia. IEEE; 2018. P. 0473-0478.

[19] Hui Liu, Houshang Darabi, Pat Banerjee, Jing Liu. Survey of Wireless Indoor Positioning Techniques and Systems. IEEE Transactions on Systems, Man, and Cybernetics. 2007; 37(6): 1067-1080.

[20] Khalel, Ayad MH. Position location techniques in wireless communication systems. Digitala Vetenskapliga Arkivet. 2010; 1(1): 53

[21] Bensky, Alan. Wireless positioning technologies and applications. $2^{\text {nd }}$ Edition. London: Artech House; 2016. 1-393.

[22] P. Series. Effects of building materials and structures on radiowave propagation above about 100 MHz. Recommendation ITU-R. 2015; 1(1) :2040-1. 
[23] Aditep Chaisang, Sathaporn Promwong. Indoor localization distance error analysis with UWB wireless propagation model using positioning method. 2018 International Conference on Digital Arts, Media and Technology (ICDAMT); 2018; Phayao, Thailand. IEEE; 2018. P. 254-257.

[24] Suppachai Monta, Sathaporn Promwong, Vongkeo Kingsakda. Evaluation of ultra wideband indoor localization with trilateration and min-max techniques. 2016 13th International Conference on Electrical Engineering/Electronics, Computer, Telecommunications and Information Technology (ECTI-CON); 2016; Chiang Mai, Thailand. IEEE; 2016. P. 1-4. 